\title{
Análise Epidemiológica de Câncer de Trato Geniturinário na Região do ABC Paulista
}

\author{
Epidemiological Analysis of Genitourinary Tract Cancer in ABC Paulista Region \\ Isabela Mazzeo Turcatto ${ }^{1}$, Pedro Mastrocinque Pereira Ferreira ${ }^{1}$, André Akira Ramos Takahashi ${ }^{1}$, Stefanie de Sousa Antunes Alcantara ${ }^{1}$, \\ Laércio da Silva Paiva², Luiz Vinicius de Alcantara Sousa²
}

Como citar: Turcatto IM, Ferreira PMP, Takahashi AAR, Alcantara SSA, Paiva LS, Sousa LVA. Análise Epidemiológica de Câncer de Trato Geniturinário na Região do ABC Paulista. Clin Onc Let. 2020; Ahead of Print. https://doi.org/10.4322/col.2019.002

\begin{abstract}
RESUMO
INTRODUÇÃO: Os cânceres do trato geniturinário foram responsáveis por 2,26\% das mortes mundiais em 2017. Entre as neoplasias mais comuns estão a de bexiga, próstata e colo de útero. MÉTODO: Estudo observacional, com avaliação temporal de 2008 a 2017, referente às admissões hospitalares por câncer de próstata, colo de útero e bexiga no ABC Paulista, São Paulo e Sudeste. Os dados secundários foram obtidos no banco de dados do DATASUS. A Classificação Internacional das doenças (CID10) foi utilizada, sendo o câncer de próstata C61, colo de útero C53 e bexiga C67. Para análise das variáveis utilizou-se Regressão Linear, com os valores de ß (coeficiente) e $r^{2}$ (capacidade preditiva do modelo), e intervalo de confiança de 95\%. RESULTADOS: A taxa de internação por cânceres geniturinários em homens aumentou no $A B C$ Paulista ( $(3: 41$ p: 0,002), São Paulo ( $(2,62, p:<0,001)$ e Sudeste ( $(2,36, p:<0,001)$. Em mulheres, a taxa de internação apresentou aumento no Grande $A B C(B: 0,93, p: 0,002)$ e em São Paulo ( $(3: 0,23, p:<0,064)$. No Sudeste, houve redução da taxa (B: -0,18, p: 0,034). CONCLUSÃO: A incidência de internações por cânceres geniturinários aumentou nas três regiões para ambos os sexos, com exceção da população feminina do Sudeste.
\end{abstract}

Palavras-chave: câncer, geniturinário, epidemiologia, bexiga, próstata e colo de útero

\begin{abstract}
INTRODUCTION: Genitourinary cancer were responsible for 2,26\% of global deaths in the2017. Among then, the most common were urinary bladder neoplasm, prostate neoplasm and cervical cancer. METHOD: An observational study, with temporal evaluation from 2008 to 2017, referring to hospital admission for prostate, cervical and urinary bladder cancer in Southeast brazilian's region, ABC Paulista and São Paulo. The secondary data were obtained into DATASUS. The International Classification of Disease (ICD 10) were used to classifies prostate cancer (C61), cervical cancer (C53) and urinary bladder neoplasm (C67). To analyze the obtained data, the study used a Linear Regression, with $\beta$ (coefficient) and $r^{2}$ (predictive model value) values, and a trust interval of 95\%. RESULTS: The hospital admission rates raised in both $A B C$ Paulista ( $(3,41$ p: 0,002), São Paulo (ß: 2,62, p: <0,001) and Southeast ( $ß: 2,36, p:<0,001)$ for male

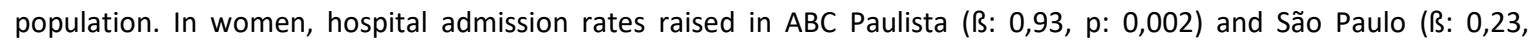
$p:<0,064)$. Meanwhile, the Southeast's rate reduced in females ( $B:-0,18, p: 0,034)$. CONCLUSION: The incidence of hospital admission from geniturinary tract cancers raised in the three regions analysed in this study, except for female population in Southeast.
\end{abstract}

Keywords: cancer, genitourinary, epidemiology, urinary bladder, prostate and cervix uteri

\section{INTRODUÇÃO}

No ano de 2017, 17,04\% do total de mortes mundial foi causada por câncer, sendo que os cânceres do trato geniturinário foram responsáveis por 2,26\% desse valor, segundo dados do Global Burden Disease (GBD, 2017). ${ }^{1}$ Em 2018, 9.6 milhões de pessoas morreram de câncer, segundo estatísticas da Organização Mundial da Saúde $(\mathrm{WHO})^{2}$

\footnotetext{
1. Centro Universitário Saúde ABC, Santo André, SP, Brasil.

2. Laboratório de Epidemiologia e Análises de dados. Centro Universitário Saúde ABC, Santo André, SP, Brasil.

Financiamento: Não se aplica.

Conflito de interesse: Os autores declararam não haver conflitos de interesse que precisam ser informados.
} 
São considerados cânceres do trato genitourinário os tumores urológicos, que acometem rim, pelve renal, ureter, bexiga, uretra, próstata, pênis, testículo, escroto e córtex adrenal, esse último incluído na 8o edição da Classificação de Tumores Maligno, ${ }^{3-5}$ e tumores ginecológicos, como corpo do útero, colo do útero, ovário, tubas uterinas, vagina, vulva e tumores trofoblásticos gestacionais. ${ }^{5}$

Os cânceres urológicos de rim, bexiga e próstata apresentaram, em 2013, 2,1 milhões de casos no mundo, cerca de 2,5 vezes mais do que no ano de $1990 .{ }^{6}$ O câncer de próstata é o mais comum entre os tumores urológicos, com 164690 novos casos nos Estados Unidos da América em 2018, seguido pelo câncer de bexiga, que ocupa o quarto lugar nas neoplasias mais comuns em homens e o nono nas mulheres, segundo a University of California San Francisco Health (UCSF, 2018), e teve incidência, em 2018, de 81190 casos. $^{7}$ O câncer de colo de útero, por sua vez, é o quarto mais comum na população feminina mundial, o que corresponde a $6,6 \%$ de todos os cânceres em mulheres no ano de 2018 , sendo que a maioria das mortes ocorre em países de média e baixa renda. ${ }^{2}$

O câncer de bexiga é um dos mais comuns do trato urinário, com 430 mil novos casos em $2012,{ }^{8}$ e possui grande relação com fatores de exposição, entre eles o tabaco e os hidrocarbonetos aromáticos policíclicos. ${ }^{9} \mathrm{O}$ estado de São Paulo é o terceiro estado brasileiro com mais mortes pela neoplasia, tendo 0,46\% do número de mortes do estado causado pelo carcinoma de bexiga, de acordo com o GBD. ${ }^{1}$

Nos homens, o câncer de próstata é o segundo mais prevalente do mundo, atrás apenas do carcinoma de pele não-melanoma. ${ }^{10}$ No Brasil, o câncer de próstata é o segundo mais comum nos homens, sendo 60.180 novos casos em 2012, 61.200 em 2016 e 68.220 em 2018. ${ }^{10,11}$ A maioria desses homens foi diagnosticado nas regiões mais desenvolvidas, sendo a região Sudeste e a região Nordeste os locais em que o câncer de próstata é o mais incidente entre os homens. ${ }^{8}$ Em relação à mortalidade, o câncer de próstata sucede o câncer de pulmão como causa de morte mais comum entre homens brasileiros, ${ }^{10}$ sendo estimado cerca de 13 mil mortes por ano devido à neoplasia. ${ }^{11}$

Nas mulheres, o câncer cervical é um importante problema de saúde público. É mais comum em mulheres de baixo nível socioeconômico, com atividade sexual precoce e múltiplos parceiros. Mundialmente, foram 266.000 mortes em 2012 , sendo que $87 \%$ das mortes ocorre em países menos desenvolvidos. ${ }^{12}$ No Brasil, foram diagnosticados 16.370 novos casos em 2018 , correspondendo a 8,1\% dos cânceres em mulheres. ${ }^{13}$

Pela significativa incidência dos cânceres geniturinários em homens e mulheres em nível mundial e nacional, esse estudo tem como objetivo analisar a incidência dos canceres de próstata, colo de útero e bexiga na região de São Paulo e do ABC.

\section{MÉTODO}

\section{DESENHO DE ESTUDO E POPULAÇÃO}

Estudo observacional de caráter ecológico, com avaliação temporal baseada em dados secundários referentes a admissões hospitalares por câncer de próstata, colo de útero e bexiga da região do Grande $A B C$, São Paulo e região Sudeste nos anos de 2008 a 2017.

$O$ estudo foi realizado com os indivíduos residentes da região do $A B C$, que possui 2.702 .071 de pessoas segundo Instituto Brasileiro de Geografia e Estatística (IBGE, 2015). A região faz parte da área metropolitana de São Paulo, e inicialmente, formada pelas cidades de Santo André, São Bernardo do Campo e São Caetano do Sul, foi dado o nome de $A B C$, mas atualmente é comum serem incorporadas as cidades de Diadema, Mauá, Ribeirão Pires e Rio Grande da Serra. $O$ Grande $A B C$ é uma importante região industrial do Estado de São Paulo, por ser berço de indústrias automobilísticas e multinacionais e por constituir um grande mercado consumidor, além de representarem uma parte importante no Produto Interno Bruto (PIB) do estado de São Paulo. ${ }^{14}$

\section{COLETA DE DADOS}

As definições de neoplasia maligna de próstata, neoplasia maligna de colo de útero e neoplasia maligna de bexiga basearam-se na décima revisão da Classificação Internacional das Doenças (CID10), correspondendo, respectivamente, aos códigos: $\mathrm{C} 61, \mathrm{C} 53$ e $\mathrm{C} 67$. 
O número de admissões hospitalares foi obtido pelo Sistema de Informação Hospitalar do Sistema Único de Saúde (SIH/SUS), disponível no DATASUS, plataforma do Ministério da Saúde Brasileiro que recebe, valida e divulga dados oficiais sobre a saúde pública do Brasil. A plataforma tem acesso livre ao usuário no site http://datasus.saude.gov.br/.

A coleta de dados na plataforma obedeceu aos códigos da CID-10. Também foram considerados durante a coleta o sexo e a idade dos pacientes internados no período pré-estabelecido, estratificação etária utilizada: 20-24, 25-39, 30-34, 35-39, 40-44, 45-49, 50-54, 55-59, 60-64, 65-69, 70-74, 75-79 anos. Todos os critérios de coleta foram obedecidos para cada uma das regiões estabelecidas para o estudo, sendo que, foram utilizadas as subdivisões da Região de Saúde de São Paulo, entre elas Grande ABC e São Paulo, e região Sudeste do Brasil.

Os dados referentes à população total do Brasil utilizados nesse estudo foram obtidos pelos censos de 2000-2010 do Instituto Brasileiro de Geografia e Estatística. (IBGE). Os censos são decenais, e, no intervalo entre eles, é feita uma estimativa para obter valores anuais das características populacionais.

Para o cálculo da taxa de internação, foi realizada a divisão entre o número de internações e a população da região em determinado ano e faixa etária, e, em seguida, a multiplicação desse valor por 100 mil. Por fim, realizou-se a correção para nível de comparação. ${ }^{15,16}$

\section{VARIÁVEIS DO ESTUDO}

As variáveis de interesse estudadas foram: número e incidência de admissões hospitalares por câncer de próstata, do colo de útero e bexiga na região do $A B C$, na cidade de São Paulo e na região Sudeste.

\section{ANÁLISE ESTATÍSTICA}

Foi avaliada a tendência da taxa internação hospitalar por câncer de próstata, colo de útero e bexiga utilizando-se a Regressão Linear (valores de $\beta$ - coeficiente e $r^{2}$ - capacidade preditiva do modelo). ${ }^{16,17} \mathrm{O}$ nível de confiança adotado foi de $95 \%$ e o Data Analysis and Statistical Software for Professionals (Stata) versão $13.0^{\circledR}$ foi o programa estatístico utilizado.

\section{RESULTADOS}

O número total de internações por cânceres geniturinários, incluindo homens e mulheres, entre os anos de 2008 e 2017, foi de 7.644 no ABC Paulista, 33.823 em São Paulo e 253.794 na região Sudeste. Quando comparados a população total e a incidência da internação do respectivo local, a média dos anos estudados é de $0,043 \%$ no $A B C$, $0,043 \%$ em São Paulo e 0,046\% no Sudeste (Tabela 1).

Tabela 1. Incidência dos cânceres geniturinários segundo as regiões estudadas.

\begin{tabular}{cccc}
\hline Ano & Região do ABC & Cidade de São Paulo & Sudeste \\
\hline 2008 & $0,023 \%$ & $0,029 \%$ & $0,038 \%$ \\
2009 & $0,028 \%$ & $0,037 \%$ & $0,041 \%$ \\
2010 & $0,038 \%$ & $0,042 \%$ & $0,044 \%$ \\
2011 & $0,043 \%$ & $0,041 \%$ & $0,044 \%$ \\
2012 & $0,050 \%$ & $0,045 \%$ & $0,046 \%$ \\
2013 & $0,047 \%$ & $0,045 \%$ & $0,047 \%$ \\
2014 & $0,047 \%$ & $0,049 \%$ & $0,047 \%$ \\
2015 & $0,048 \%$ & $0,047 \%$ & $0,050 \%$ \\
2016 & $0,051 \%$ & $0,046 \%$ & $0,049 \%$ \\
2017 & $0,050 \%$ & $0,050 \%$ & $0,053 \%$ \\
\hline
\end{tabular}

Classificação internacional de doenças, 10a revisão. Códigos: C61, C53 e C67. Dados Populacionais: Instituto Brasileiro de Geografia e Estatística. Censos 2000 e 2010. Fonte: Sistema de Informações Hospitalares (SIH / SUS). Dados disponibilizados pelo Departamento de Informática do Sistema Nacional de Saúde (DATASUS - www.datasus.gov.br). Ministério da Saúde, Brasil. 
A região que apresentou maior aumento das taxas de internação masculinas foi o Grande $A B C(\beta: 3,41, p: 0,002)$. $O$ aumento da taxa internações masculinas nessa região predominou nas faixas etárias acima de 55 anos ( $\beta: 27,05$, p: 0,019), sendo que o maior aumento foi em homens de 65 a 69 anos $(\beta: 89,67, p:<0,001)$ (Tabela 2). São Paulo $(\beta: 2,62, p:<0,001)$ também teve predomínio a partir dos 55 anos e maior aumento entre 65-69 anos $(\beta: 75,78$, $\mathrm{p}:<0,001)$, com significância presente em todas as idades a partir dos 55 anos (Tabela 2). A região Sudeste ( $\beta: 2,36$, $\mathrm{p}:<0,001)$, obteve significância a partir dos 45 anos, apesar do maior aumento assemelhar-se aos dados etários do Grande ABC e São Paulo, ou seja, predomínio a partir dos 55 anos e com maior índice de aumento entre 65-69 anos ( $\beta: 65,67, p:<0,001$ ) (Tabela 2). Portanto, nas três localizações estudadas, os cânceres geniturinários mostraram-se mais incidentes em idades mais avançadas, com pico aos 65 anos, o que foi confirmado com o intervalo de confiança considerado significante $(p<0,05)$. Além do aumento significante a partir dos 55 anos na região do Grande $A B C$, houve um aumento significante dos 20 aos 24 anos ( $\beta: 6,9, p: 0,031)$ (Tabela 2). Na região Sudeste, homens de 25 a 29 anos apresentaram redução significante ( $\beta$ : 0,26, p: 0,033) (Tabela 2$)$.

Tabela 2. Regressão linear da incidência* de hospitalização (por 100.000 habitantes) do sexo masculino entre o período de 2000 e 2010.

\begin{tabular}{|c|c|c|c|c|c|c|}
\hline \multirow{2}{*}{ Faixa Etária } & \multicolumn{2}{|c|}{ Região do ABC } & \multicolumn{2}{|c|}{ Cidade de São Paulo } & \multicolumn{2}{|c|}{ Sudeste } \\
\hline & $\boldsymbol{\beta}$ & p* & $\beta$ & $p^{*}$ & $\beta$ & p* \\
\hline $20-24$ & 6,9 & 0,031 & $-0,03$ & 0,169 & $-0,004$ & 0,976 \\
\hline $25-29$ & 3,22 & 0,306 & $-0,62$ & 0,218 & $-0,26$ & 0,033 \\
\hline $30-34$ & $-0,7$ & 0,657 & $-0,03$ & 0,938 & 0,089 & 0,47 \\
\hline $35-39$ & $-1,78$ & 0,356 & 0,41 & 0,667 & 0,55 & 0,075 \\
\hline $40-44$ & $-1,43$ & 0,632 & 0,39 & 0,772 & 0,25 & 0,55 \\
\hline $45-49$ & 3,012 & 0,409 & 4,33 & 0,093 & 3,56 & 0,001 \\
\hline $50-54$ & 4,35 & 0,565 & 5,46 & 0,309 & 7,31 & 0,008 \\
\hline $55-59$ & 27,05 & 0,019 & 29,83 & 0,002 & 25,27 & $<0,001$ \\
\hline $60-64$ & 81,02 & 0,001 & 57,33 & $<0,001$ & 51,67 & $<0,001$ \\
\hline $65-69$ & 89,67 & $<0,001$ & 75,78 & $<0,001$ & 65,67 & $<0,001$ \\
\hline $70-74$ & 75,73 & 0,009 & 55,22 & 0,003 & 47,39 & $<0,001$ \\
\hline $75-79$ & 64,24 & 0,027 & 35,14 & 0,003 & 34,93 & $<0,001$ \\
\hline
\end{tabular}

Padronizado para a idade de acordo com a população mundial da Organização Mundial da Saúde. Classificação internacional de doenças, 10a revisão. Códigos: C61, C53 e C67. * Regressão. $\beta$ : valor de beta da regressão. Fonte: Sistema de Informações Hospitalares (SIH / SUS). Dados disponibilizados pelo Departamento de Informática do Sistema Nacional de Saúde (DATASUS - www.datasus.gov.br). Ministério da Saúde, Brasil.

As taxas de internação feminina sofreram aumento no Grande $A B C(\beta: 0,93, p: 0,002)$ (Tabela 3) e em São Paulo $(\beta: 0,23, p:<0,064)$ (Tabela 3), porém, esse aumento não se mostrou significante em São Paulo. A região Sudeste apresentou uma redução da taxa de internação $(\beta: 0,18, p: 0,034)$ (Tabela 3). No Grande $A B C$, a faixa etária com maior aumento é de 55-59 anos ( $\beta$ : 17,25, p: 0,001), porém, as faixas etárias de 20-24, 30-34, 40-44 e 50-54 anos também apresentaram aumentos significantes nas taxas de cânceres geniturinários (Tabela 3). Em São Paulo, diferentemente do Grande ABC, o aumento foi maior em mulheres de 65 a 69 anos ( $\beta: 7,64, p: 0,021)$, mas também houve aumento significante na faixa etária de 35 a 39 anos, além de uma redução dos 20 aos 24 anos ( $\beta$ : $-0,21$, p: 0,031) (Tabela 3). No Sudeste, mulheres de 55 a 79 anos apresentaram aumento significante de internações por câncer, com predomínio entre 60 a 64 anos $(\beta: 6,84, p:<0,001)$ (Tabela 3). Porém, ainda na região Sudeste, as faixas etárias de 20-24, 40-44, 45-49 e 50-54 anos apresentaram redução significativa $(p<0,05)$ nas internações, sendo essa redução maior em mulheres de 45 a 49 anos ( $\beta$ : -15,31, p: <0,001) (Tabela 3). 
Tabela 3. Regressão linear da incidência* de hospitalização (por 100.000 habitantes) do sexo feminino entre o período de 2000 e 2010 .

\begin{tabular}{|c|c|c|c|c|c|c|}
\hline \multirow{2}{*}{ Faixa Etária } & \multicolumn{2}{|c|}{ Região do ABC } & \multicolumn{2}{|c|}{ Cidade de São Paulo } & \multicolumn{2}{|c|}{ Sudeste } \\
\hline & $\boldsymbol{\beta}$ & $\mathbf{p}$ & $\beta$ & $\mathbf{p}$ & $\beta$ & $\mathbf{p}$ \\
\hline $20-24$ & 6,9 & 0,031 & $-0,21$ & 0,031 & $-2,28$ & 0,018 \\
\hline $25-29$ & 3,22 & 0,306 & $-0,04$ & 0,973 & $-1,68$ & 0,204 \\
\hline $30-34$ & 6,06 & 0,033 & $-1,52$ & 0,502 & $-2,34$ & 0,166 \\
\hline $35-39$ & 11,05 & 0,062 & 6,21 & 0,021 & $-0,36$ & 0,85 \\
\hline $40-44$ & 12,56 & 0,052 & $-1,32$ & 0,377 & $-12,72$ & $<0,001$ \\
\hline $45-49$ & 3,56 & 0,327 & 0,03 & 0,992 & $-15,31$ & $<0,001$ \\
\hline $50-54$ & 10,66 & 0,027 & $-1,81$ & 0,446 & $-4,35$ & 0,02 \\
\hline $55-59$ & 17,25 & 0,001 & 4,94 & 0,144 & 4,1 & 0,019 \\
\hline $60-64$ & 9,93 & 0,203 & 6,17 & 0,078 & 6,84 & $<0,001$ \\
\hline $65-69$ & $-1,75$ & 0,742 & 7,64 & 0,021 & 4,3 & 0,004 \\
\hline $70-74$ & 6,29 & 0,328 & 1,96 & 0,401 & 2,22 & 0,03 \\
\hline $75-79$ & 8,12 & 0,155 & 3,35 & 0,09 & 3,18 & 0,003 \\
\hline
\end{tabular}

Padronizado para a idade de acordo com a população mundial da Organização Mundial da Saúde. Classificação internacional de doenças, 10a revisão. Códigos: C61, C53 e C67. * Regressão. $\beta$ : valor de beta da regressão. Fonte: Sistema de Informações Hospitalares (SIH / SUS). Dados disponibilizados pelo Departamento de Informática do Sistema Nacional de Saúde (DATASUS - www.datasus.gov.br). Ministério da Saúde, Brasil.

\section{DISCUSSÃO}

O predomínio das doenças crônicas não transmissíveis sobre as doenças transmissíveis é um problema de saúde pública a ser enfrentado mundialmente. Por isso, estudos referentes à epidemiologia dessas doenças têm assumido grande significância, com objetivo de proporcionar melhorias ao sistema de saúde através de programas de prevenção, rastreamento, diagnóstico e tratamento que atinjam todas as populações de forma eficaz.

Os resultados encontrados nesse estudo mostraram um aumento no número de cânceres geniturinários nas regiões de São Paulo, ABC e Sudeste, dado compatível com outros estudos, que apresentaram resultados com aumento no número de casos mundiais de cânceres urológicos, tendo como fatores que justificam esse aumento o crescimento populacional, aumento da expectativa de vida, aumento de índices de mortalidade, evolução de técnicas diagnósticas, relacionados à melhora no acesso a programas de prevenção, métodos diagnósticos e tratamentos. ${ }^{6,18}$ Esses dados foram corroborados por estudos que somaram os fatores com um melhor acesso a dados epidemiológicos. ${ }^{10,11}$

Porém, em 2018, um estudo apresentou resultados contrários ao desse estudo ao registrar uma redução de $2 \%$ ao ano de câncer em homens da população norte-americana, com o câncer de próstata apresentando redução de $10 \%$ ao ano entre 2010 e $2014 .^{7}$

O aumento de internações por câncer esteve presente para ambos os sexos, exceto na população feminina da região Sudeste. Esse aumento foi maior em homens, o que pode ser explicado pelo câncer de próstata ter representado, no ano de 2012, 15\% dos cânceres diagnosticados nos homens. ${ }^{10,11}$ Desses, 60.180 casos ocorreram no Brasil, principalmente na região Sudeste, devido ao maior desenvolvimento e acesso à saúde para diagnóstico precoce, e maior abrangência da campanha "Novembro Azul", que visa, conscientizar os homens em relação à saúde. ${ }^{10,19}$

Além disso, a maior exposição aos fatores de risco também explica esse aumento. Estudo classificou os fatores de risco conhecidos para câncer de bexiga de acordo com o grau de evidência científica apresentado por cada um..$^{20}$ Entre os fatores de risco com evidência comprovada estão a idade (>45 anos), sexo masculino, fumo de cigarro, exposição ocupacional à aminas aromáticas e hidrocarbonetos aromáticos policíclicos, arsênio, medicações, como fenacetina, ciclofosfamida e clorpromazina, radiação e fatores genéticos. ${ }^{20}$

O tabagismo, relacionado com o uso precoce e à dose diária, é fator de risco para as neoplasias geniturinárias, como câncer de colo de útero e o carcinoma de bexiga, uma vez que os carcinógenos presentes no cigarro induzem danos ao DNA que colaboram para a carcinogênese. ${ }^{21,22}$ Apesar das campanhas antitabagistas presentes no Brasil 
desde 1985 e intensificadas em 2005, houve aumento das internações demonstrado nesse estudo, que pode ser explicado pela alta prevalência de fumantes uma vez que a carcinogênese depende dos anos de exposição prévia à fatores de risco, entre eles o tabaco. ${ }^{23,24}$

A população masculina, por sua vez, pode ter a maior taxa de internação quando comparada à população feminina, explicada pelo maior consumo de tabaco. Em 1989, dos 35\% da população tabagista, 43,09\% eram do sexo masculino, contra $26,87 \%$ do sexo feminino, ${ }^{24}$ sendo que os homens expostos nesse período estão agora atingindo a idade de desenvolvimento da neoplasia, que nesse estudo foi de 55 anos para homens, com pico na 60 década de vida, e de 65 a 70 anos em outros estudos. ${ }^{25}$

Apesar da grande relação das neoplasias com o tabagismo, estudo realizado no Reino Unido, ${ }^{26}$ mostra que $7,1 \%$ dos cânceres de bexiga em homens e 1,9\% em mulheres tem a exposição ocupacional às aminas aromáticas, hidrocarbonetos aromáticos policíclicos e outras substâncias químicas presentes nas indústrias de pinturas, borracha e alumínio, como fator de risco. ${ }^{20} \mathrm{Com}$ isso, por ser o $\mathrm{ABC}$ uma região industrial, também poderia explicar a população dessa região estar sujeita a uma maior exposição aos fatores de risco e, consequentemente, a um maior índice de internações por cânceres.

Além disso, a discrepância de incidência entre homens e mulheres também pode ser explicada pelos hormônios sexuais androgênicos e estrogênicos, atividade metabólica enzimática e intensidade da avaliação diagnóstica. 20,25,27

Por sua vez, o câncer de colo de útero costuma ser mais prevalente em países menos desenvolvidos, tendo ocupado, em 2014, o 4ㅇ lugar de mortes causadas por neoplasias em mulheres brasileiras. ${ }^{12,28}$ O Brasil acompanha os dados mundiais, tendo menor incidência do câncer nas regiões mais desenvolvidas do país, como o Sudeste $\left(9,97 / 100\right.$ mil) e a região Sul $\left(14,07 / 100\right.$ mil), ambas regiões com maior e melhor acesso à saúde. ${ }^{13}$ Assim, a existência de programas para prevenção, como a vacinação, uso de preservativo, ações educativas, rastreamento, feito através do exame citopatológico em mulheres sexualmente ativas de 25 anos a 64 anos, diagnóstico e tratamento mais eficazes, corroboram com os resultados presentes nesse estudo, que mostraram uma redução na internação feminina na região Sudeste. ${ }^{21,28}$

Os dados etários encontrados nesse estudo assemelham-se aos encontrados em outros estudos, ${ }^{21}$ de modo que, o aumento nas internações femininas apresentado a partir da 40 década de vida pode ser explicado pelo incentivo à realização do exame citopatológico anualmente. Porém, o aumento a partir da 4으 década foi maior na região do $A B C$, enquanto a cidade de São Paulo apresentou aumento significante apenas em mulheres maiores de 65 anos, e a região Sudeste, cuja realização do exame deve abranger uma parcela populacional maior, apresentou redução significante na 40 década de vida, seguida de aumento significante nas 5 e 6을écadas.

O Papilomavírus Humano (HPV), principalmente os subtipos 16 e 18, que são considerados os de potencial oncogênico mais alto, é um importante fator de risco para o desenvolvimento do câncer de colo de útero, principalmente em casos de infecção persistente, quando os tipos oncogênicos do vírus se instalam, causando lesões celulares pré-cancerosas seguidas por invasão, ${ }^{29}$ que costumam ocorrer em mulheres que adquirem o vírus em idade mais avançada, explicando o maior número de internações de mulheres nessa faixa etária encontrado nesse estudo. $^{21}$

Porém, as campanhas de vacinação contra o vírus disponíveis no Sistema Único de Saúde (SUS) tanto para meninas quanto para meninos, não podem justificar as reduções de internação em mulheres apresentadas nesse estudo por serem de início recente, de modo que a população vacinada não atingiu a faixa etária de pico de desenvolvimento das neoplasias geniturinárias, ou seja, a população entre 9 e 13 anos, vacinada antes do início da vida sexual e consequente exposição ao vírus, deve atingir a 4ㅇ, 5ㅇ e 6을écada de vida para que sejam contabilizadas possíveis reduções devido ao menor número de mulheres infectadas..$^{21,28,30}$

Apesar de recente, alguns estudos já avaliaram a eficácia da vacina. Uma revisão da literatura mostrou que após a implementação da vacina houve redução das infeç̧ões e verrugas genitais causadas pelos genótipos do vírus inclusos na vacina, assim como a redução de lesões pré-cancerosas. ${ }^{30}$ 


\section{CONCLUSÃO}

A incidência de internações por cânceres geniturinários aumentou tanto no Grande $A B C$, quanto em São Paulo e no Sudeste, com exceção da população feminina do Sudeste. Esse aumento foi maior em homens, porém, para ambos os sexos as faixas etárias de maior aumento foram na 5 e 6음 década de vida.

\section{REFERENCIAS}

1. The Global Burden of Disease. Institute for Health Metrics and Evaluation [Internet]. Seattle: Global Burden of Disease; 2017 [citado 2019 fev 15]. Disponível em: https://vizhub.healthdata.org/gbd-compare/

2. Bray F, Ferlay J, Soerjomataram I, Siegel RL, Torre LA, Jemal A. Global cancer statistics 2018: GLOBOCAN estimates of incidence and mortality worldwide for 36 cancers in 185 countries. CA Cancer J Clin. 2018;68(6):394-424. http://dx.doi.org/10.3322/caac.21492. PMid:30207593.

3. World Health Organization. Cancer country profile 2014 [Internet]. Genebra: WHO; 2014 [citado 2019 jul 7]. Disponível em: https://www.who.int/cancer/country-profiles/bra_en.pdf?ua=1

4. Bukowski RM. Genitourinary oncology: current status and future challenges. Front Oncol. 2011;1:32. http://dx.doi.org/10.3389/fonc.2011.00032. PMid:22649760.

5. Universidade Estadual de Campinas. Diretrizes baseadas em evidências em tumores urológicos [Internet]. 7. ed. Campinas: UNICAMP; 2015 [citado 2019 jul 7]. Disponível em: https://www.fcm.unicamp.br/fcm/sites/default/files/2016/page/manual_uroonco_2015.pdf

6. Dy GW, Gore JL, Forouzanfar MH, Naghavi M, Fitzmaurice C, Catto J. Global burden of urologic cancers, 1990-2013. Eur Urol. 2017;71(3):437-46. http://dx.doi.org/10.1016/j.eururo.2016.10.008. PMid:28029399.

7. Siegel RL, Miller KD, Jemal A. Cancer statistics, 2018. CA Cancer J Clin. 2018;68(1):7-30. http://dx.doi.org/10.3322/caac.21442. PMid:29313949.

8. Instituto Nacional de Câncer José Alencar Gomes da Silva. Estimativa 2012: incidência do câncer no Brasil. Rio de Janeiro: INCA; 2012.

9. Cha JD, Lourenço DB, Korkes F. Analysis of the association between bladder carcinoma and arsenic concentration in soil and water in southeast Brazil. Int Braz J Urol. 2018;44(5):906-13. http://dx.doi.org/10.1590/s16775538.ibju.2017.0543. PMid:30044600.

10. Tourinho-Barbosa RR, Pompeo ACL, Glina S. Prostate cancer in Brazil and Latin America: epidemiology and screening. Int Braz J Urol. 2016;42(6):1081-90. http://dx.doi.org/10.1590/s1677-5538.ibju.2015.0690.

11. Sasse AD, Wiermann EG, Herchenhorn D, et al. First Brazilian consensus of advanced prostate cancer: recommendations for clinical practice. Int Braz J Urol. 2017;43(3):407-15. http://dx.doi.org/10.1590/s16775538.ibju.2016.0490. PMid:28199075.

12. Viscondi JYK, Faustino CG, Campolina AG, Itria A, Soárez PC. Simple but not simpler : a systematic review of Markov models for economic evaluation of cervical cancer screening. Clinics. 2018;73:1-12.

http://dx.doi.org/10.6061/clinics/2018/e385. PMid:29995100.

13. Instituto Nacional de Câncer José Alencar Gomes da Silva. Estimativa 2018: incidência do câncer no Brasil. Rio de Janeiro: INCA; 2018.

14. Consórcio Intermunicipal Grande ABC. O ABC [Internet]. Santo André; 2019 [citado 2019 maio 30]. Disponível em: http://consorcioabc.sp.gov.br/o-grande-abc

15. Ahmad OB, Boschi-Pinto C, Lopez AD, et al. Age standardization of rates: a new WHO standard. Geneva: WHO; 2001.

16. Sousa LVA, Paiva LDS, Figueiredo FWDS, Almeida TCDC, Oliveira FR, Adami F. Trends in stroke-related mortality in the ABC region, São Paulo, Brazil: an ecological study between 1997 and 2012. Open Cardiovasc Med J. 2017;11(1):111-9. http://dx.doi.org/10.2174/1874192401711010111. PMid:29290833. 
17. Silva Paiva L, Oliveira FR, Alcantara Sousa LV, Santos Figueiredo FW, Sá TH, Adami F. Decline in stroke mortality between 1997 and 2012 by sex: ecological study in brazilians aged 15 to 49 years. Sci Rep. 2019;9(1):2962. http://dx.doi.org/10.1038/s41598-019-39566-8. PMid:30814591.

18. Soria F, Shariat SF, Lerner SP, et al. Epidemiology, diagnosis, preoperative evaluation and prognostic assessment of upper-tract urothelial carcinoma (UTUC). World J Urol. 2017;35(3):379-87. http://dx.doi.org/10.1007/s00345-016-1928-x. PMid:27604375.

19. Modesto AAD, Lima RLB, D’Angelis AC, Augusto DK. Um novembro não tão azul: Debatendo rastreamento de câncer de próstata e saúde do homem. Interface Commun Heal Educ. 2018;22(64):251-62. http://dx.doi.org/10.1590/180757622016.0288 .

20. Malats N, Real FX. Epidemiology of Bladder Cancer. Hematol Oncol Clin North Am. 2015;29(2):177-89, vii. http://dx.doi.org/10.1016/j.hoc.2014.10.001. PMid:25836927.

21. Thuler LCS, Bergmann A, Casado L. Perfil das pacientes com câncer do colo do útero no Brasil, 2000-2009: estudo de base secundária. Rev Bras Cancerol. 2012;58(3):351-7. http://dx.doi.org/10.32635/2176-9745.RBC.2012v58n3.583.

22. Jin F, Thaiparambil J, Donepudi SR, et al. Tobacco-specific carcinogens induce hypermethylation, DNA adducts, and DNA damage in bladder cancer. Cancer Prev Res. 2017;10(10):588-97. http://dx.doi.org/10.1158/1940-6207.CAPR-170198. PMid:28851690.

23. Instituto Nacional de Câncer José Alencar Gomes da Silva. Programa Nacional de Controle do Tabagismo. Tratamento do tabagismo [Internet]. Rio de Janeiro: INCA; 2019 [citado 2019 jul 7]. Disponível em:

https://www.inca.gov.br/programa-nacional-de-controle-do-tabagismo\#1

24. Iglesias R, Jha P, Pinto M, Silva VLDC, Godinho J. Controle do tabagismo no Brasil [Internet]. Washington: The World Bank; 2007 [citado 2019 jul 15]. Disponível em: http://actbr.org.br/uploads/arquivo/202_controle-tabagismo-brasilBM.pdf

25. Kirkali Z, Chan T, Manoharan M, et al. Bladder cancer: epidemiology, staging and grading, and diagnosis. Urology. 2005;66(6, Suppl 1):4-34. http://dx.doi.org/10.1016/j.urology.2005.07.062. PMid:16399414.

26. Brown T, Slack R, Rushton L. Occupational cancer in Britain. Urinary tract cancers: bladder and kidney. Br J Cancer. 2012;107(Suppl 1):S76-84. http://dx.doi.org/10.1038/bjc.2012.121. PMid:22710682.

27. Dobruch J, Daneshmand S, Fisch M, et al. Gender and bladder cancer: a collaborative review of etiology, biology, and outcomes. Eur Urol. 2016;69(2):300-10. http://dx.doi.org/10.1016/j.eururo.2015.08.037. PMid:26346676.

28. Brasil. Ministério da Saúde. Secretaria de Vigilância em Saúde. Departamento de Vigilância de Doenças Transmissíveis. Coordenação-Geral do Programa Nacional de Imunizações. Informe Técnico sobre a Vacina Papilomavírus Humano (HPV) na Atenção Básica [Internet]. Brasília: Ministério da Saúde; 2014 [citado 2019 jul 15]. Disponível em: https://portalarquivos2.saude.gov.br/images/pdf/2015/junho/26/Informe-T--cnico-Introdu----o-vacina-HPV-18-22014.pdf

29. Goodman A. HPV testing as a screen for cervical cancer. BMJ. 2015;350(1):1-11. http://dx.doi.org/10.1136/bmj.h2372. PMid:26126623.

30. Maver PJ, Poljak M. Progress in prophylactic human papillomavirus (HPV) vaccination in 2016: a literature review. Vaccine. 2018;36(36):5416-23. http://dx.doi.org/10.1016/j.vaccine.2017.07.113. PMid:28801154. 